\title{
Multi-Objective Drag and Sound Optimization of Square Cylinder Retrofitted with Splitter Plate
}

\author{
Ramesh Babu S., Karthik K., Jeyakumar S.
}

\begin{abstract}
The application of a splitter plate on square cylinders to reduce drag in cross-flow is an active research area. However, the associated aeroacoustic sound in the surrounding fluid also undergoes reduction, an aspect which has received less attention. This paper presents a numerical methodology for coupled aerodynamic and aeroacoustic calculations for flow past square cylinders with various splitter plate lengths. Computations are performed at a Re of 22000 using URANS technique and $\mathrm{FW}-\mathrm{H}$ analogy. Observing that drag reduction and sound reduction occur at different splitter plate lengths, a two-objective optimization problem is posed to minimize the drag and OASPL simultaneously.
\end{abstract} Particle swarm optimization.

\section{INTRODUCTION}

The interaction of high-speed flow past a solid body generates unsteady fluid dynamic loads, which in turn produces flow-induced noise [1]. Aerodynamic loads and the associated aeroacoustic sound emitted from the bluff bodies are of concern for many engineering applications, such as bullet train, aircraft landing gear, automobiles etc. [2]-[6]. As, the requirements for the environmental noise emission levels of aircraft and automobiles, driven by societal demand [7], are becoming more stringent, it is important to understand and control these flow-induced problems so that engineering design and public comfort can be improved continuously. The aerodynamic drag force exerted on high-speed vehicles is very much associated with the engine power and consequently the fuel expenditure [8], [9]. So, the aerodynamic drag force exerted on the solid body and the sound radiated to the surroundings is of foremost concern in many industrial applications [10]-[12]. Therefore, the numerical computation of aerodynamic and aeroacoustic characteristics of rigid bluff bodies have received extensive attention [13]-[15].

The development of aerodynamics led to streamlined structures which have decreased aerodynamic drag and self-induced noise in comparison to bluff bodies [16], [17].

Revised Manuscript Received on December 05, 2019.

* Correspondence Author

Ramesh Babu S., Ramesh Babu S, Department of Mechanical Engineering, Kalasalingam Academy of Research and Education, Krishnankoil, Srivilliputhur, Tamil Nadu 626128, rbabu0223@gmail.com.

Karthik K.*, Department of Aeronautical Engineering, Kalasalingam Academy of Research and Education, Krishnankoil, Srivilliputhur, Tamil Nadu 626128.karthik@klu.ac.in.

Jeyakumar S., Department of Aeronautical Engineering, Kalasalingam Academy of Research and Education, Krishnankoil, Srivilliputhur, Tamil Nadu 626128, sjeyakumar1974@gmail.com.
Keywords: Aeroacoustics, Aerodynamics, Passive control,

This is attributable to the retarded separation of the boundary layer, decreased wake region and decreased vortex generation [18]. But most structures encountered in the engineering applications are bluff bodies which include bridge piers, buildings, vehicles, chimneys, cooling towers, heat exchanger tubes, flame-holders, aircraft undercarriage during landing, pipelines and re-entry vehicles, to mention a few. Hence studying the aerodynamics and aeroacoustics of bluff bodies, has been the field of interest of several numerical and experimental investigations [19]-[27]. From the literature survey, it is observed that most of the analysis is concentrated on circular cylinders and a few reported investigations are found on the study of aerodynamic drag and flow-induced noise on flows over the square cylinder[26], [28]-[32]. The air flow over a square cylinder and the associated aerodynamics is significant because the flow separation point is permanent. But, in the case of a circular cylinder, the flow separation point alters periodically around the aft of the cylinder. So, the case study selected for this paper is a square cylinder.

The flow-control techniques mainly fall into two groups namely, active and passive [33]-[35]. The active flow control technique needs a secondary energy provider and a monitoring method [36], but the passive flow control techniques are easy to execute, and the method involves a comprehensive parametric study [37]. Due to their simplicity and no external power requirement in their mechanism, passive control techniques are preferred over active control techniques [38]. The flow over a circular cylinder retrofitted with splitter plate is a popular passive flow control method that has received much attention in recent times [39]-[48]. The literature review shows that the presence of a splitter plate was found to lessen the aerodynamic force exerted on the cylinder, and the aeroacoustic sound radiated to the surroundings. A bluff body attached with a downstream flat plate changes the behaviour of the near wake structure and followed by the aerodynamic characteristics. Numerical analysis carried out by [45], [47] for flow over a circular cylinder attached with splitter plate found that there exists a critical length of the splitter plate where the complete suppression of aerodynamic drag is obtained.

Despite the promising previous works on the circular cylinder attached with splitter plate, published results of the flow-induced sound of square cylinder retrofitted with splitter plate are scarce [32], [49]. The possibility for a downstream flat plate to be applied in sound cancellation mechanism for square cylinder was proven by Doolan [50]. That study

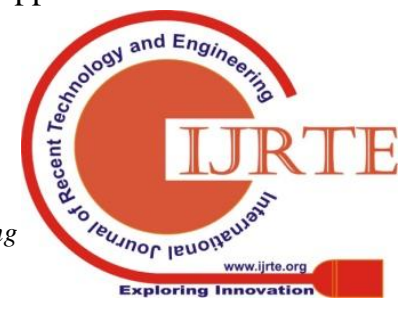


found that the plate fundamentally changed the flow structure of the cylinder wake, which was beneficial to a significant reduction in the Strouhal number and total drag coefficient of the square cylinder. However, an extended study is needed to investigate whether similar phenomena can be obtained splitter plate of various lengths. Ali et al. [32] numerically investigated the sound emitted from the square cylinder retrofitted with a splitter plate at low Reynolds number $(\mathrm{Re}=150)$ without incidence for various lengths $(0.5 \mathrm{D}-6 \mathrm{D})$. A sound pressure level decrement of $3 \mathrm{~dB}$ was attained when the splitter plate length was equal to $1 \mathrm{D}$. Ogunremi and Sumner [49] studied the usefulness of the presence of a splitter plate for the square cylinder, experimentally, at $\operatorname{Re}=74000$. The study found that a splitter plate of $\mathrm{L} / \mathrm{D} \geq 3$ was sufficient to reduce the drag and suppress vortex shedding. In summary, only a few studies have been performed for computing both the aerodynamic drag and aeroacoustic sound of cylinders with a splitter. Hence, the current study aims to investigate the influence of a square cylinder with a splitter plate on the flow-induced sound and aerodynamic drag in much more detail. Almost all studies with splitter plates either focused on aerodynamic drag or the aeroacoustic sound emission at low Re. In most industrial applications, however, the aerodynamic sound becomes more significant only for high Re flows. Hence, in this paper, a high $\operatorname{Re}(=22000)$ flow is treated. The current study has two objectives. First, to examine how the splitter plate lengths affect the aerodynamic drag and sound. Second, to locate the optimal length to reduce the drag and sound simultaneously.

In this paper, a two-dimensional parametric study is employed; where the unsteady Reynolds-averaged NavierStokes (URANS) technique has been utilized for aerodynamic calculations and Ffowcs Williams-Hawking's acoustic analogy [51] has been used for aeroacoustics calculations for the considered problem. A total of 10 splitter plate lengths have been analyzed to find the optimal splitter plate length that could minimize the aerodynamic drag force and flow-induced noise simultaneously. In other words, a multi-objective unconstrained optimization problem is posed where mean aerodynamic drag coefficient (CD, mean) and overall sound pressure level (OASPL) are the two objective functions to be minimized in ' $\mathrm{L} / \mathrm{D}$ ' design parameter space. To identify these two optimal shape parameters, particle swarm optimization (PSO) method [52], 53] has been adopted using a radial basis neural network (RBNN) [54], [55] with L/D as a design variable. It should be noted that the PSO technique is extensively used in various engineering applications, specifically, the method has also been applied to flow-induced sound reduction [45], [55], [56].

The organization of the article is arranged as follows. The geometry and flow specifications are briefly described in section 2 followed by the computational methodology in Section 3. The obtained numerical results are discussed in Section 4, concentrating on the impact of splitter plate length on the CD, mean and OASPL. Lastly, the conclusions drawn from our study are provided in Section 5.

\section{GEOMETRY AND FLOW CONDITIONS}

In this work, square cylinders retrofitted with 10 different splitter plate lengths of thickness 0.02Dare considered (Fig. $1)$. The normalized splitter plate lengths are varied in the range of $\mathrm{L} / \mathrm{D}=0.5$ to 5 at $0.5 \mathrm{D}$ intervals because these ranges were found effective in passive flow control at subcritical $\operatorname{Re}[49]$. The normalized thickness of the rear plate is $0.02 \mathrm{D}[32]$. All simulations in this paper are carried out at $\mathrm{Re}$ $=\rho U D / \mu=22000$, where $\rho$ is the density of air $(=1.225$ $\mathrm{kg} / \mathrm{m} 3), \mathrm{U}$ is the incoming flow velocity $(=32 \mathrm{~m} / \mathrm{s}), \mathrm{D}$ is the side length of cylinder $(=0.01 \mathrm{~m})$ and $\mu$ is the dynamic viscosity of air $(=1.7894 \times 10-5$ Pa.s $)$. Figure 1 shows the schematic of the square cylinder with a splitter plate placed at the downstream location.

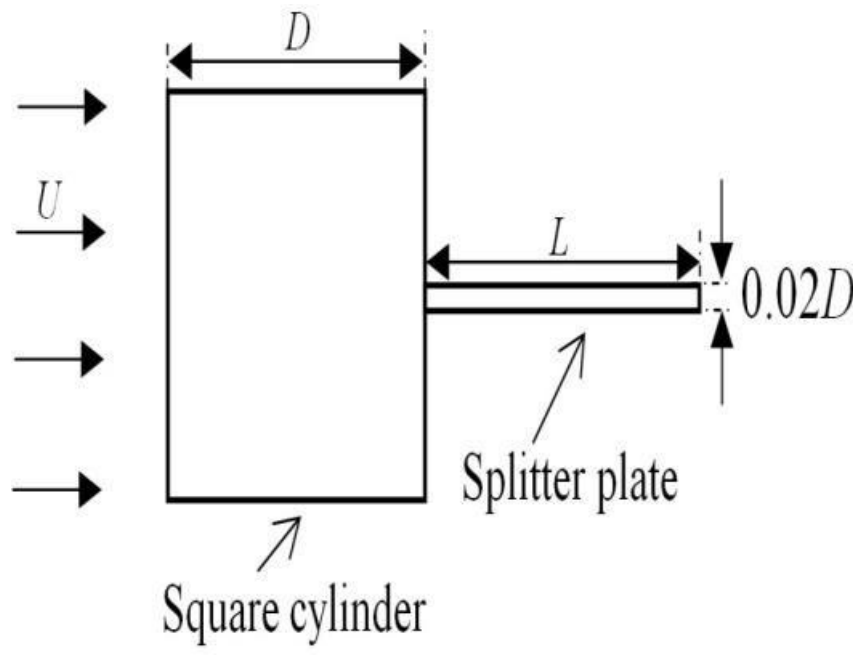

Figure 1: Definition sketch

\section{COMPUTATIONAL METHODOLOGY}

The turbulent air flow field over the cylinder is computed by utilizing the two-dimensional unsteady Reynolds averaged Navier Stokes (URANS) technique [31], where the numerical model simultaneously solves the mass and momentum conservation equations. Even though the flow field in this problem is three-dimensional in nature, the two-dimensional method can, however, obtain the significant aerodynamic and aeroacoustic characteristics [20], [31], [57] at a cheap computational expense and is a more appropriate technique for a parametric study [58]. The k- $\omega$ SST model [59], [60] is employed to provide closure to the system of equations formed by URANS. The finite volume method with the QUICK scheme [61] is utilized for the spatial discretization, while a first-order implicit scheme is used for temporal discretization. The SIMPLE algorithm is utilised for the pressure-velocity coupling. An adaptive time-stepping method is used in the paper, where the time steps are adjusted automatically with respect to the truncation error [45]. The time-step is allowed to change in between 10-8 s and 10-4 s. When the residuals of all variables fall just below 10-5, the solution has been regarded as converged. To compute the aerodynamic drag coefficient and acoustic pressure signals, the unsteady information is collected, once the fluid flow

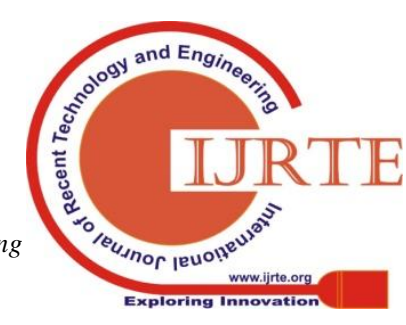


attains the statistically steady-state. The flow- induced sound is calculated using the FW-H acoustic analogy [51] with the two-dimensional URANS results as input by using a "correlation length" method [20], [57].

The "correlation length" technique presumes that the vortex shedding is completely correlated over a certain length of the cylinder in the lengthwise direction [19]. The correlation length that is required in this method can be found experimentally by determining the surface static pressure along the length of the cylinder. In this paper, a correlation length of 4D is adopted based on the previous experimental data [62], [63]. Detailed explanations of the fluid governing equations, acoustic analogy and correlation length model may be found in previous studies [19], [24], [31]. The CFD simulations have been carried out by implementing the cylinder models in the commercial flow solver ANSYS Fluent, and a MATLAB code was developed for processing the acoustic pressure data.

In this work, two-objective optimization was carried out to simultaneously minimize the aerodynamic drag force and the flow-induced sound of the cylinder retrofitted with a splitter plate. The flowchart in Fig. 2 illustrates the optimization process used in this study. The design variables and objective functions are defined, and the design variables are randomly chosen from the sample space. For reducing computational expense, the RBNN model [54], [55] is employed in this work to evaluate the objective functions from a finite set of design variables for which the objective functions are evaluated numerically. For searching optimal values, the PSO algorithm is used, which is based on the work of Kennedy and Eberhart [53], by introducing the nondominated solution concept [52]. The Multi-objective particle swarm optimization coupled with the RBNN is performed by an algorithm implemented in MATLAB code.

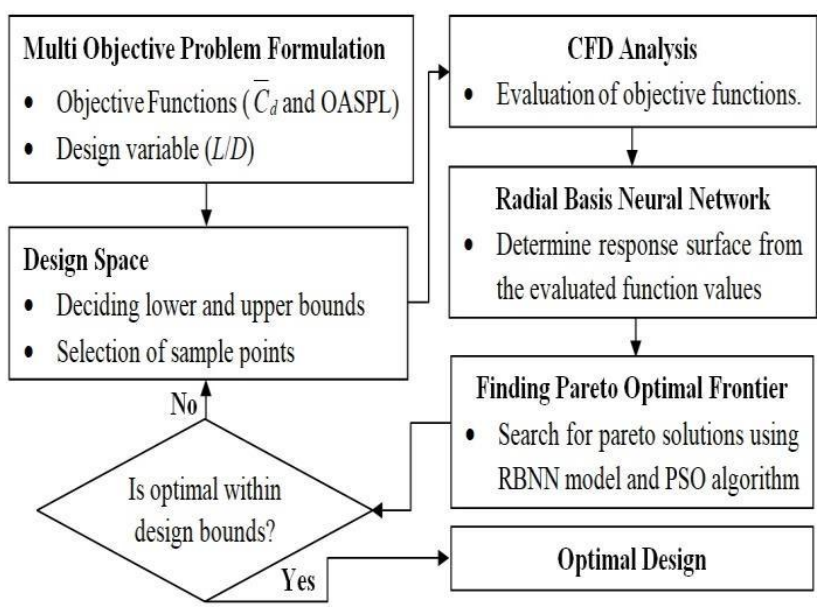

Figure 2: Design methodology.

\section{RESULTS AND DISCUSSIONS}

The dimensions of the computational domain around the two-dimensional square cylinder are shown in Fig. 3. The uniform fluid stream is normal to the inlet boundary ' $\mathrm{AB}$ ', i.e. $\mathrm{X}$-axis is parallel to the incoming velocity $\mathrm{U}$. The origin $(0,0)$ of the coordinate system is positioned at the midpoint of the cylinder. The boundaries ' $\mathrm{BC}$ ' and ' $\mathrm{AD}$ ' have zero shear stress and normal velocity. The outlet boundary ' $\mathrm{CD}$ ' has a zero value of gauge pressure. The no-slip (wall) condition is assigned on the cylinder surface. The computational domain size is chosen as $31.5 \mathrm{D} \times 21 \mathrm{D}$ [31].

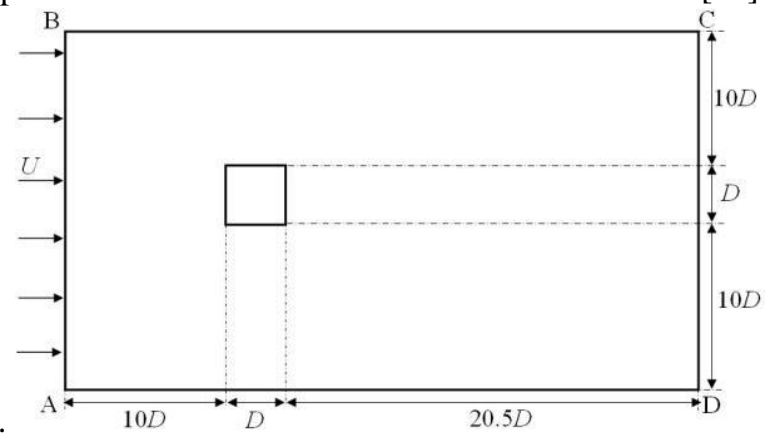

Figure 3: Schematic diagram of the computational
domain

Structured O-grids with circumferential clustering in the vicinity of the square cylinder are provided and the remaining computational domain is meshed with fine unstructured triangular grid system (Fig. 4). A near wall resolution of $\mathrm{y}+=1$ has been preserved near the cylinder surface for fully resolving the laminar sublayer. The number of O-grids in the radial direction is 20 , and the first cell distance from the cylinder surface is $7.1 \times 10-6 \mathrm{~m}$ with a growth ratio of 1.2. Along the peripheral surface of the cylinder, the cells are being spaced equally at $0.02 \mathrm{D}(=\Delta \mathrm{s})$ apart. The accuracy of numerical results is reliant on mesh resolution, so a mesh sensitivity test is conducted in this study. The rms lift coefficient (CL, rms), OASPL (refer eq. 2) and sound pressure level (SPL, refer eq. 1) spectrum of three grid system are chosen for mesh dependency test, and are presented in Table 1 and Fig 5. It can be observed from these results that the computational domain with1.22 lakh cells (Case 3) is adequate for the current study. Limited by the computational sources, in the present work, the mesh dependency check is conducted only for the unmodified cylinder. Since all cylinders retrofitted with splitter plate operate at same $\operatorname{Re}$ as the unmodified cylinder, it is reasonable to accept that the grid convergence of bare cylinder will be same for cylinders with splitter plate also [32].

Table 1: Summarized results of the grid sensitivity test

\begin{tabular}{|l|l|l|l|l|}
\hline Case & Cells (Lakhs) & $\Delta s$ & $C_{L, r m s}$ & OASPL(dB) \\
\hline 1 & 1.16 & $0.04 D$ & 1.504 & 77.91 \\
\hline 2 & 1.18 & $0.03 D$ & 1.503 & 77.76 \\
\hline 3 & 1.22 & $0.02 D$ & 1.477 & 77.46 \\
\hline
\end{tabular}




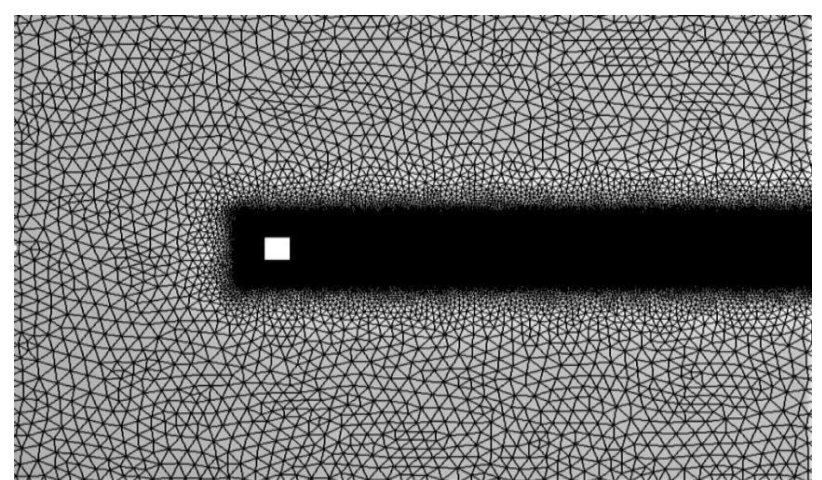

Fig.4.(a) Computational mesh Full view

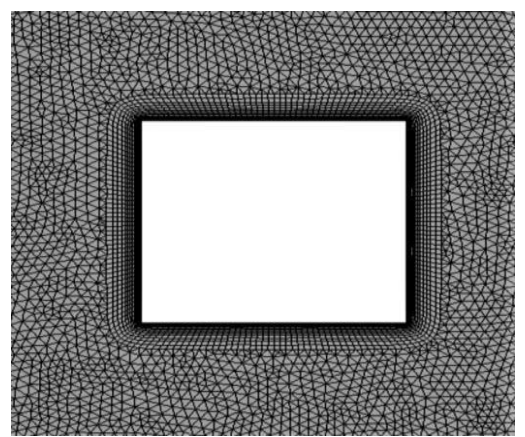

Figure 4:b) Computational mesh near view.

Table 1: Summarized results of the grid sensitivity test

\begin{tabular}{|c|c|c|c|c|}
\hline Case & Cells (Lakhs) & $\Delta s$ & $C_{L, r m s}$ & OASPL(dB) \\
\hline 1 & 1.16 & $0.04 D$ & 1.504 & 77.91 \\
\hline 2 & 1.18 & $0.03 D$ & 1.503 & 77.76 \\
\hline 3 & 1.22 & $0.02 D$ & 1.477 & 77.46 \\
\hline
\end{tabular}

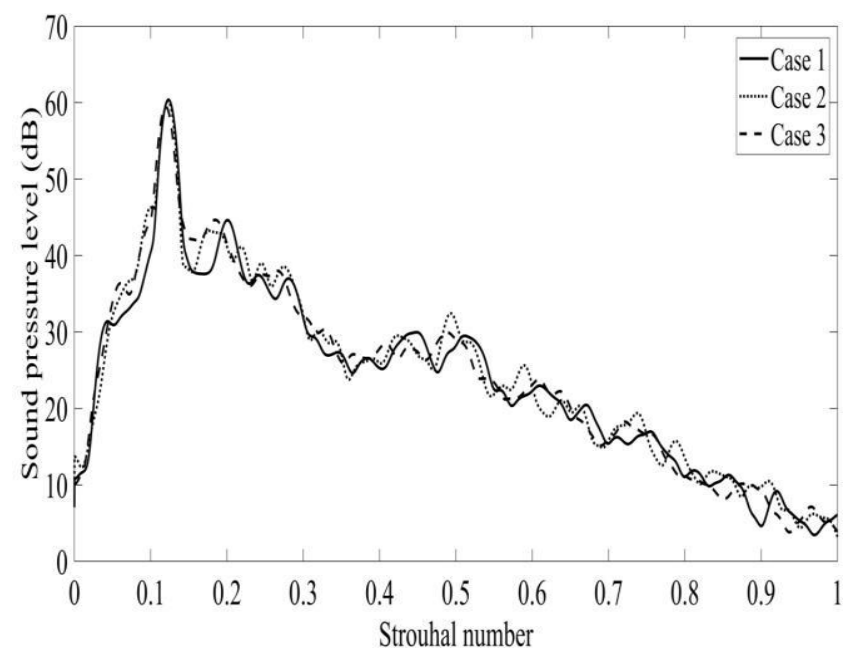
parameter.

B. Validation of aerodynamic and aeroacoustic parameters

For numerical calculations, the turbulent airflow stream should attain a statistically steady state and this takes place at approximately $0.1 \mathrm{~s}$. In all computations, the entire simulation time is $1.1 \mathrm{~s}$ and the final $1 \mathrm{~s}$ (i.e. from $0.1 \mathrm{~s}$ to 1.1 s) of time history data is used in calculating the aerodynamic and acoustic results. In other words, flow sampling occurred
Figure 5: Grid sensitivity study using SPL as a

over a non-dimensional time of $\mathrm{tU} / \mathrm{D}=3200$, which equates to approximately 384 vortex-shedding cycles. The precision of the SPL calculations primarily experimental results by Iglesias et al. [29]. The SPL spectrum reported by Iglesias et al. [29] is for $\operatorname{Re} 54,800$, which is scaled to $\operatorname{Re} 22,000$ by using the acoustic scaling method proposed by Moreau and Doolan [27]. The sound measurements were conducted in an open jet anechoic wind tunnel on a square cylinder. The power spectral density of the calculated sound pressure at the receiver location has been obtained using Welch technique[70] adopting Hann window with 50\% overlap of 32 data segments [71]. The SPL (dB) is defined as relies on the accuracy of the aerodynamic solution. To validate this solution, the mean aerodynamic drag coefficient (CD,mean), rms lift coefficient (CL,rms) and Strouhal number (St) corresponding to the dominant vortex shedding frequency, computed numerically, are compared with previous studies (Table 2). The aerodynamic forces on the cylinder consist of two main parts: pressure and shear force acting in directions normal and tangential to the cylinder surface, respectively. The pressure and shear forces exerting on the cylinder surface are integrated, and resolved in the $x-$ and $y$ directions to obtain the drag and lift forces, respectively. The $\mathrm{CD}$, mean, CL,rms and St of the cylinder obtained from simulations are in good agreement with the previous studies(Table 2). The St corresponding to the dominant vortex shedding frequency is computed by fast Fourier transform (FFT) of the lift force time history (Fig. 6).

Table 2: Comparison of the numerical solution with literature

\begin{tabular}{|l|l|l|l|l|}
\hline Author & $\begin{array}{l}\text { Method } \\
\text { (Author) }\end{array}$ & $\boldsymbol{C}_{\boldsymbol{D}, \text { mean }}$ & $\boldsymbol{C}_{\boldsymbol{L}, \boldsymbol{r m s}}$ & $\boldsymbol{S t}$ \\
\hline Present & $k-\omega$ SST & 2.183 & 1.477 & 0.12 \\
\hline Tian et al. [64] & $k-\omega$ SST & 2.06 & 1.492 & 0.138 \\
\hline $\begin{array}{l}\text { Samion et al. } \\
\text { [31] }\end{array}$ & $k-\omega$ SST & 2.1 & 1.43 & 0.126 \\
\hline $\begin{array}{l}\text { Bosch and } \\
\text { Rodi [65] }\end{array}$ & $k-\varepsilon$ & 2.108 & 1.012 & 0.146 \\
\hline $\begin{array}{l}\text { Shimada and } \\
\text { Ishihara [66] }\end{array}$ & $k-\varepsilon$ & 2.05 & 1.43 & 0.141 \\
\hline $\begin{array}{l}\text { Murakami and } \\
\text { Mochida [67] }\end{array}$ & LES & 2.09 & 1.6 & 0.132 \\
\hline Sohankar [68] & LES & 2.19 & 1.433 & 0.118 \\
\hline Park [69] & Experiment & 2.1 & - & 0.13 \\
\hline Vickery [63] & Experiment & 2.044 & 1.299 & 0.12 \\
\hline
\end{tabular}

Figure 7 displays the comparison of the computed SPL spectrum with experimental data. The computed spectrum agrees with the experimental spectrum at the principal regions and shows an adequate match elsewhere. The most important characteristics of this spectrum, specifically, tonal Strouhal number (i.e. the Strouhal number associated with the peak frequency of the spectrum, represented by StT) and the tonal SPL at this frequency (represented by SPLT in $\mathrm{dB}$ ) match precisely

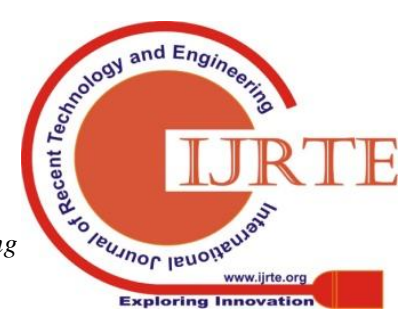


with experimental results as shown in Table 3. This means that the URANS and FW-H method utilized in this study is capable to predict the important features of the flow induced sound, and also has the capability to present additional insight through a parametric study. To make evident the sound reduction levels at the receiver location, the OASPL, which is an estimate of the total sound energy of the SPL spectrum is utilized in this paper. Based on the calculation using Eq. (2), the OASPL is found to be $77.46 \mathrm{~dB}$.

To evaluate the accuracy of the far-field acoustic analysis, the computed flow-induced sound data are validated using experimental data. Comparing the StT(see Table 3) with the St(see Table 2), it is clear that the peak sound frequency takes place at the dominant vortex shedding frequency, which means the dominant role of vortex shedding on aeroacoustic sound generation. So,

by amending the vortical flow structures in the cylinder wake, the sound production can be controlled [43] and this is what a cylinder retrofitted with a splitter plate does.

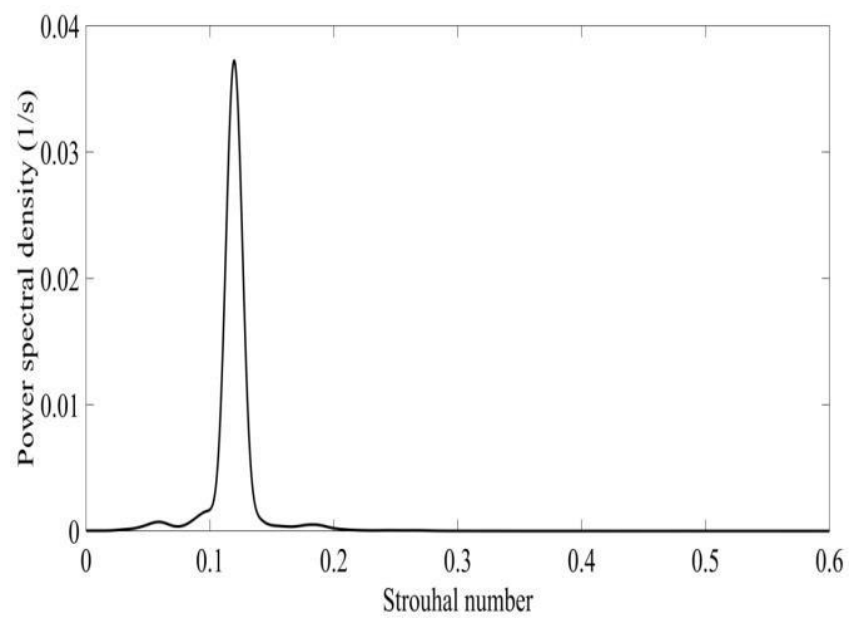

Figure 6: Power spectral density of the lift coefficient

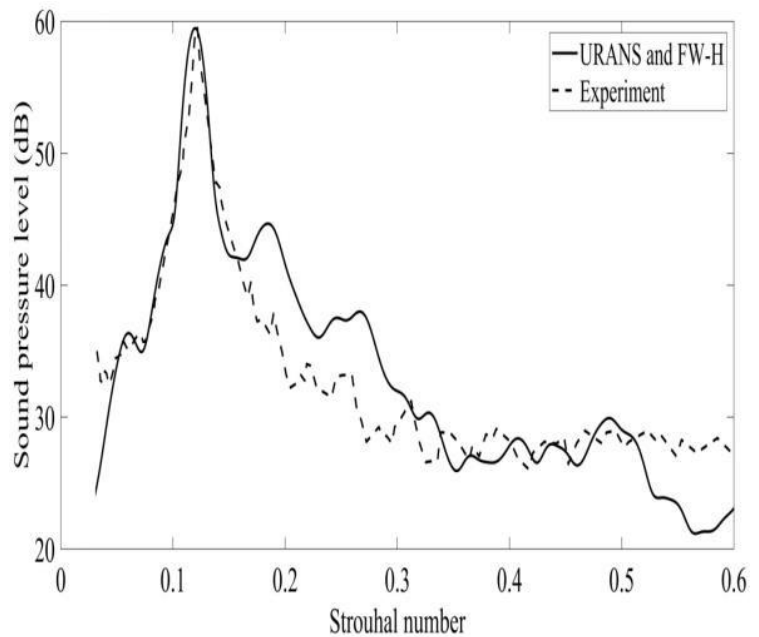

Figure 7: Comparison of the numerical SPL spectrum with the experimental result.

Table 3: Comparison of the calculated sound parameters with experimental results.

\begin{tabular}{|l|l|l|}
\hline Acoustic parameters & Exp. & Num. \\
\hline
\end{tabular}

\begin{tabular}{|l|l|l|}
\hline$S t_{T}$ & 0.122 & 0.12 \\
\hline $\mathrm{SPL}_{\mathrm{T}}(\mathrm{dB})$ & 59.57 & 59.46 \\
\hline
\end{tabular}

In the present work, ten square cylinders attached with splitter plate models with different L/D, also called the 'design variables' in the context of optimisation (see Fig. 2), and are simulated numerically. The instantaneous drag force and flow-induced sound pressure of all the models were numerically computed. Table 4 records the values of $\mathrm{CD}$, mean and OASPL for all cases. It can be noticed that the mean drag force (CD, mean) and flow-induced sound (represented by OASPL) of cylinders with splitter are significantly suppressed, with respect to the unmodified cylinder. This is due to the increased base pressure of the modified cylinder compared to the unmodified one [72]. Ogunremi and Sumner [49] found that a square cylinder attached with a splitter plate of length $\mathrm{L} / \mathrm{D}=3$ undergo additional drag decline than that of $\mathrm{L} / \mathrm{D}=2$ at subcritical $\mathrm{Re}$. The present findings are consistent with the results obtained by Ogunremi and Sumner [49]. The presence of splitter plate shows the way to a considerable decrease of the vorticity magnitude and turbulent kinetic energy [32], [43], and in turn, reduces flow-induced sound [57]. When the splitter plate length of the modified cylinder $\mathrm{L} / \mathrm{D}=3.5$, the drag and sound emission reduce by about $22.95 \%$ and $2.1 \mathrm{~dB}$, respectively. And, when $\mathrm{L} / \mathrm{D}=1$, the drag and sound emission are found to reduce by about $15.54 \%$ and $5.61 \mathrm{~dB}$, respectively (Table 4). The variations of $\mathrm{CD}$, mean and OASPL for various L/D suggest an optimization problem.

Table 4: Variation of CD, mean and OASPL with splitter plate length $(\mathrm{L} / \mathrm{D})$

\begin{tabular}{|l|l|l|l|l|}
\hline \multirow{2}{*}{$L / D$} & \multicolumn{3}{|l|}{$C_{D, \text { mean }}$} & \multicolumn{2}{l|}{ OASPL } \\
\cline { 2 - 5 } & $C F D$ & Reduction $(\%)$ & CFD & Reduction $(\mathrm{dB})$ \\
\hline $0 D$ & 2.1829 & - & 77.46 & - \\
\hline $0.5 D$ & 1.9782 & 9.38 & 75.48 & 1.98 \\
\hline $1 D$ & 1.8437 & 15.54 & 71.85 & 5.61 \\
\hline $1.5 D$ & 1.8021 & 17.44 & 74.8 & 2.66 \\
\hline $2 D$ & 1.8137 & 16.91 & 76.4 & 1.06 \\
\hline $2.5 D$ & 1.7911 & 17.95 & 76.55 & 0.91 \\
\hline $3 D$ & 1.7239 & 21.03 & 74.13 & 3.33 \\
\hline $3.5 D$ & 1.6820 & 22.95 & 75.36 & 2.1 \\
\hline $4 D$ & 1.7783 & 18.53 & 73.71 & 3.75 \\
\hline $4.5 D$ & 1.8456 & 15.45 & 75.63 & 1.83 \\
\hline $5 D$ & 1.8850 & 13.65 & 76.02 & 1.44 \\
\hline
\end{tabular}

In the framework of optimization, we require to develop an RBNN for both CD, mean and OASPL with L/Das a design variable. Table 5 shows the predicted values of $C D$, mean and OASPL using the RBNN model within the considered parameter space, and the prediction capability was compared regarding error ratio (= Predicted value/Numerical value) [73]. The goodness of fit measure, correlation coefficient (R2) for the constructed RBNN are 0.9879 for $\mathrm{CD}$, mean and 0.91 for OASPL. The statistical parameter called root mean squared error 
(RMSE) for the constructed RBNN are 0.0143 for CD, mean and 0.0441 for OASPL. The lower RMSE values show better performance, whereas higher R2 values indicate precise predictions [74]. From section 4.3, it is clear that the minimum CD, mean and OASPL occurs at different L/D. Thus, an optimal determination of the splitter plate length the solution of a two-objective optimization problem that minimizes both the objective functions ( $C D$, mean and OASPL) simultaneously.

Table 5: Computed CFD and predicted RBNN values of CD, mean and OASPL

\begin{tabular}{|l|l|l|l|l|l|l|}
\hline \multirow{2}{*}{$L / D$} & \multicolumn{4}{|l|}{$C_{D, \text { mean }}$} & \multicolumn{3}{l|}{ OASPL $(\mathrm{dB})$} \\
\cline { 2 - 7 } & $C F D$ & $\mathrm{RBNN}$ & $\begin{array}{l}\text { Error } \\
\text { ratio }\end{array}$ & $\mathrm{CFD}$ & $\mathrm{RBNN}$ & $\begin{array}{l}\text { Error } \\
\text { ratio }\end{array}$ \\
\hline $0 D$ & 2.1829 & 2.19 & 1.003 & 77.46 & 77.47 & 1.000 \\
\hline $0.5 D$ & 1.9782 & 1.97 & 0.996 & 75.48 & 75.40 & 0.999 \\
\hline $1 D$ & 1.8437 & 1.85 & 1.003 & 71.85 & 72.10 & 1.003 \\
\hline $1.5 D$ & 1.8021 & 1.82 & 1.010 & 74.8 & 74.33 & 0.994 \\
\hline $2 D$ & 1.8137 & 1.81 & 0.998 & 76.4 & 77.07 & 1.009 \\
\hline $2.5 D$ & 1.7911 & 1.78 & 0.994 & 76.55 & 75.80 & 0.990 \\
\hline $3 D$ & 1.7239 & 1.73 & 1.004 & 74.13 & 74.82 & 1.009 \\
\hline $3.5 D$ & 1.6820 & 1.71 & 1.017 & 75.36 & 74.85 & 0.993 \\
\hline $4 D$ & 1.7783 & 1.76 & 0.990 & 73.71 & 74.01 & 1.004 \\
\hline $4.5 D$ & 1.8456 & 1.83 & 0.992 & 75.63 & 75.51 & 0.998 \\
\hline $5 D$ & 1.8850 & 1.90 & 1.008 & 76.02 & 76.04 & 1.000 \\
\hline
\end{tabular}

The objective of the multi-objective optimization is to simultaneously minimize the drag force and sound emission of the modified cylinder. The optimization problem can be posed as Minimize F1 (L/D) and F2 (L/D) subject to $0 \leq \mathrm{L} / \mathrm{D} \leq$ 5 (3) Where, F1 = CD, mean and F2 = OASPL.

Using the scheme depicted in Fig. 2, the near- optimal solutions of Eq. (3) are obtained using the PSO algorithm with an initial population of 100 . The PSO algorithm yields the Pareto front (Fig. 8) consisting of non- dominated solutions. Fig. 9 shows the design variables of the solutions lying on the Pareto front of Fig. 8.

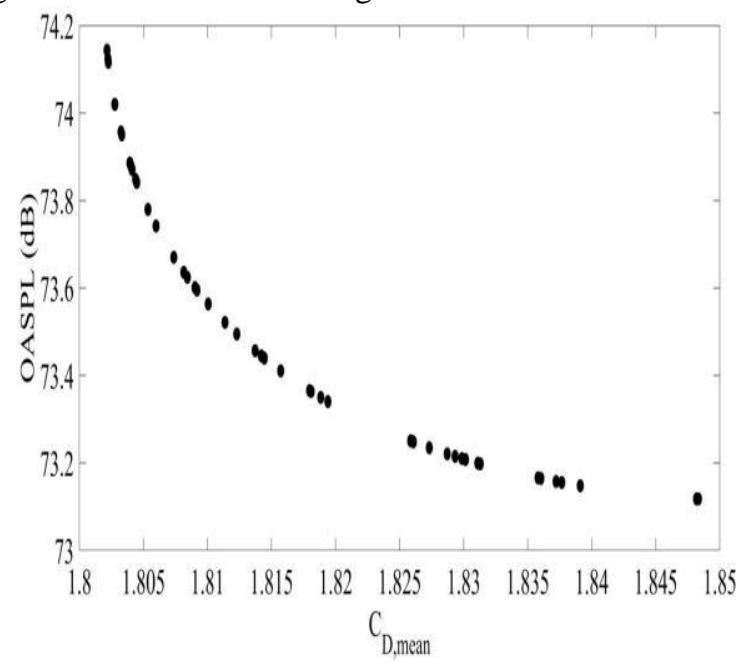

Figure 8: Pareto optimal front

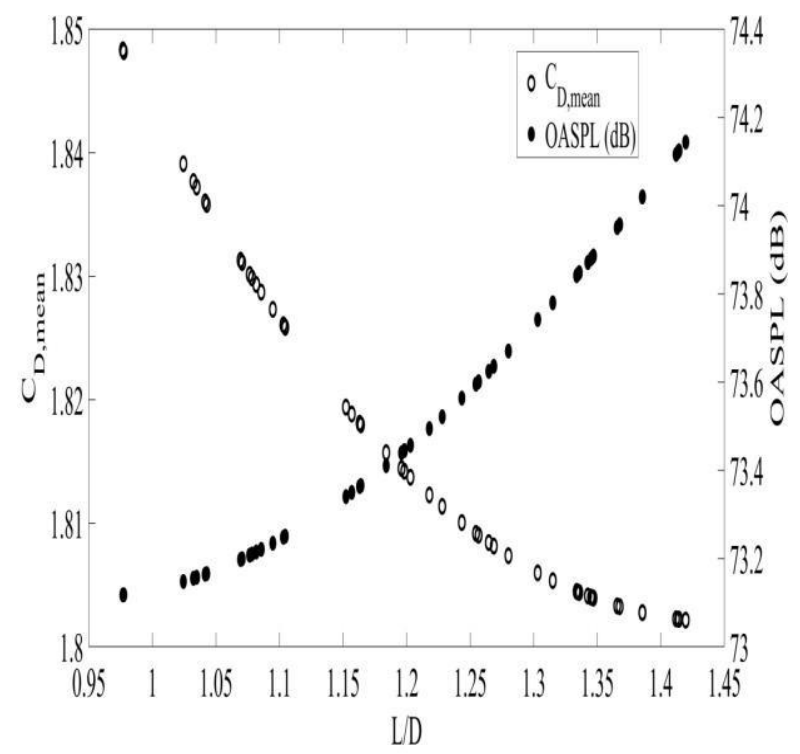

Figure 9: Pareto optimal solutions

Despite the information that the Pareto set can provide the engineer with various design solutions, a choice can be made for the 'most acceptable' solution which is the so- called 'knee point'. The 'knee point' can be found on the Pareto front using the minimum distance method [75]. The knee point $\mathrm{K}$ corresponds to minimum $\mathrm{Kj}$. Using this procedure, Eq. (4) identifies the knee point as $\mathrm{L} / \mathrm{D}=1.24$. The $\mathrm{CD}$, mean and OASPL values for the knee point, which is the 'most acceptable' solution, are given in Table 6 .

Table 6: Results for optimum configuration of the modified cylinder $(\mathrm{L} / \mathrm{D}=\mathbf{1 . 2 4})$ using $\mathrm{RBNN}$. Values in parenthesis are the difference with respect to the unmodified cylinder

\begin{tabular}{|l|l|l|}
\hline Square cylinder & $C_{D, \text { mean }}$ & OASPL $(\mathrm{dB})$ \\
\hline Unmodified & 2.1829 & 77.46 \\
\hline Modified - optimum & 1.8101 & 73.56 \\
$L / D=1.24$ & $(17.08 \%)$ & $(3.9 \mathrm{~dB})$ \\
\hline
\end{tabular}

To substantiate the reason for the reduction in $\mathrm{CD}$, mean and OASPL, a numerical simulation is done for the knee point optimum configuration. The contours obtained from the numerical simulation for the 'knee point' configuration will now be presented, always with unmodified cylinder comparison. Figures 10 and 11 shows the contours of mean averaged static pressure and the instantaneous turbulent kinetic energy, respectively, for the unmodified cylinder and the cylinder with optimal splitter plate. The pressure in the cylinder wake (i.e. base pressure) is lower for the normal cylinder, and as a result, it has a higher drag (Fig. 10) [72] The values of instantaneous turbulent kinetic energy in the near-wake (downstream) of a cylinder with optimal splitter plate are smaller (Fig. 11) [32], [43], which is the main cause for sound mitigation[57]. The results highlight that considerable improvement in aerodynamic and aeroacoustic performance of square cylinder with a splitter plate is possible as a result of 
optimization.

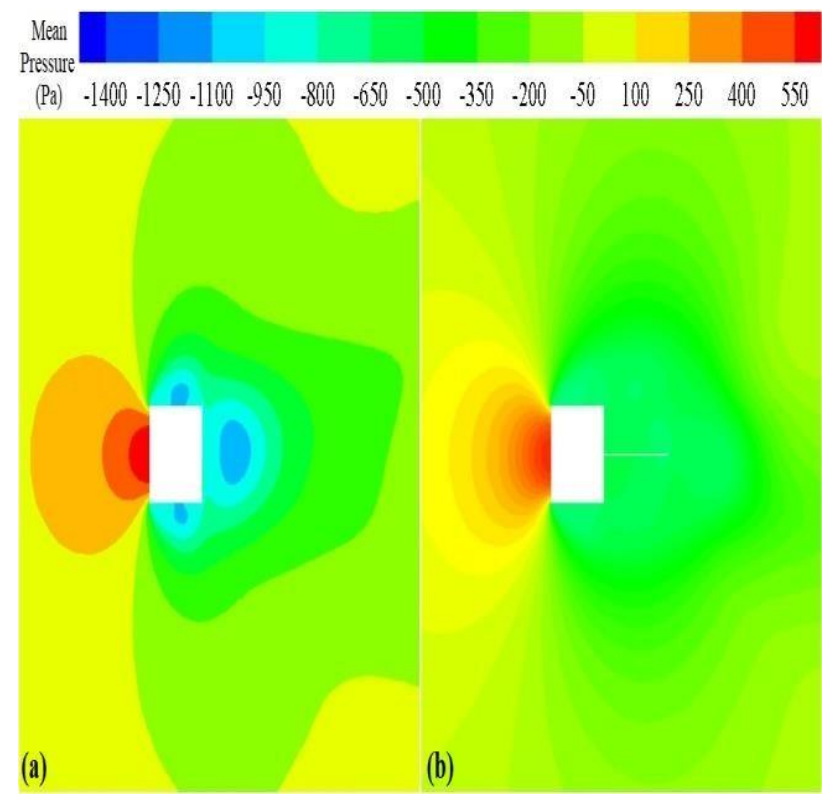

Figure 10: Mean pressure distribution around the cylinder.

(a) Unmodified, L/D = 0 (b) optimal, L/D = 1.24

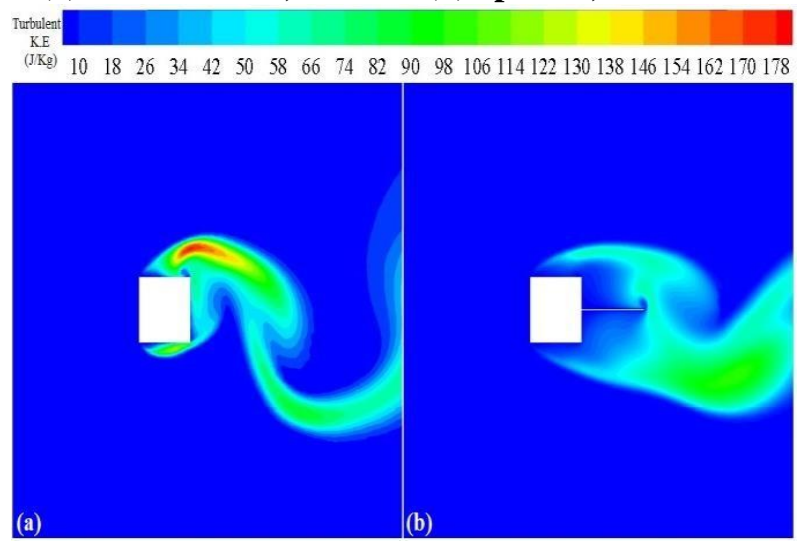

Figure 11: Instantaneous turbulent kinetic energy around the cylinder. (a) Unmodified, L/D = 0 (b) optimal, $\mathrm{L} / \mathrm{D}=\mathbf{1 . 2 4}$

\section{CONCLUSION}

The use of a square cylinder retrofitted with a splitter plate as a means to reduce drag force in cross-flow is an active area of research. However, the sound that is induced by such flows and the associated sound emission levels have not received much attention. This paper studies aerodynamic and aeroacoustic behavior of square cylinders attached with splitter plate numerically. The incoming flow field at $\mathrm{Re}=$ 22000 has been resolved using the URANS technique, and the far-field acoustic results are computed by means of the FW-H acoustic analogy. The considered ten cylinder models of various L/D show significant drag and sound mitigation. A multi-objective optimization problem is posed and numerically solved to yield a Pareto front of drag coefficient vs. OASPL using the PSO algorithm in conjunction with $\mathrm{RBNN}$. The knee point optimum if found to be $\mathrm{L} / \mathrm{D}=1.24$, and for this configuration, the drag and OASPL reduce by $17.08 \%$ and $3.9 \mathrm{~dB}$, respectively compared to the unmodified cylinder. Future work should address the behavior of cylinders of various cross-sections, with the active flow and noise control.

\section{REFERENCES}

1. S. W. Rienstra and P. Sijtsma, Review of "Aeroacoustics of low Mach number flows: Fundamentals, Analysis and Measurement," vol. 431 2018.

2. H. R. Kaviani and A. Nejat, "Aerodynamic noise prediction of a MW-class HAWT using shear wind profile," Journal of Wind Engineering and Industrial Aerodynamics, vol. 168, no. June, pp. 164-176, 2017

3. C. Talotte, "Aerodynamic Noise: a Critical Survey," Journal of Sound and Vibration, vol. 231, no. 3, pp. 549-562, 2000.

4. R. Vilela de Abreu, N. Jansson, and J. Hoffman, "Computation of aeroacoustic sources for a Gulfstream G550 nose landing gear model using adaptive FEM," Computers and Fluids, vol. 124, pp. 136-146, 2016.

5. D. Lallier-Daniels, M. Piellard, B. Coutty, and S. Moreau, "Aeroacoustic study of an axial engine cooling module using latticeBoltzmann simulations and the Ffowcs Williams and Hawkings' analogy," European Journal of Mechanics, B/Fluids, 2017.

6. M. Sanjose, S. Moreau, and J. Gullbrand, "Hybrid noise predictions of a radial notebook blower," European Journal of Mechanics, B/Fluids, 2017.

7. A. Fyhri and G. M. Aasvang, "Noise, sleep and poor health: Modeling the relationship between road traffic noise and cardiovascular problems," Science of the Total Environment, 2010.

8. Z. Mohamed-Kassim and A. Filippone, "Fuel savings on a heavy vehicle via aerodynamic drag reduction," Transportation Research Part D: Transport and Environment, 2010.

9. M. J. Rose, "Commercial vehicle fuel economy - The correlation between aerodynamic drag and fuel consumption of a typical truck," Journal of Wind Engineering and Industrial Aerodynamics, 1981.

10. A. Baron, M. Mossi, and S. Sibilla, "The alleviation of the aerodynamic drag and wave effects of high-speed trains in very long tunnels," Journal of Wind Engineering and Industrial Aerodynamics, 2001.

11. S. Khelladi, S. Kouidri, F. Bakir, and R. Rey, "Predicting tonal noise from a high rotational speed centrifugal fan," Journal of Sound and Vibration, 2008

12. R. S. Raghunathan, H. D. Kim, and T. Setoguchi, "Aerodynamics of high-speed railway train,” Progress in Aerospace Sciences, 2002.

13. K. A. Kurbatskii and R. R. Mankbadi, "Review of computational aeroacoustics algorithms," International Journal of Computational Fluid Dynamics. 2004.

14. C. K. W. Tam, "Recent advances in computational aeroacoustics," Fluid Dynamics Research, 2006.

15. M. Wang, J. B. Freund, and S. K. Lele, "COMPUTATIONAL PREDICTION OF FLOW-GENERATED SOUND," Annual Review of Fluid Mechanics, 2006.

16. P. Devinant, T. Laverne, and J. Hureau, "Experimental study of wind-turbine airfoil aerodynamics in high turbulence," Journal of Wind Engineering and Industrial Aerodynamics, 2002.

17. D. J. Moreau, Z. Prime, R. Porteous, C. J. Doolan, and V. Valeau, "Flow-induced noise of a wall-mounted finite airfoil at low-to- moderate Reynolds number," Journal of Sound and Vibration, vol. 333, no. 25, pp 6924-6941, 2014.

18. M. Wang, S. Moreau, G. Iaccarino, and M. Roger, "LES prediction of wall-pressure fluctuations and noise of a low-speed airfoil," International Journal of Aeroacoustics, vol. 8, no. 3, pp. 177-197, 2009.

19. D. Casalino and M. Jacob, "Prediction of aerodynamic sound from circular rods via spanwise statistical modelling," Journal of Sound and Vibration, vol. 262, no. 4, pp. 815-844, 2003.

20. J. S. Cox, K. S. Brentner, and C. L. Rumsey, "Computation of vortex shedding and radiated sound for a circular cylinder: Subcritical to transcritical Reynolds numbers," Theoretical and Computational Fluid Dynamics, 1998.

21. X. Gloerfelt, F. Pérot, C. Bailly, and D. Juvé, "Flow-induced cylinder noise formulated as a diffraction problem for low Mach numbers," Journal of Sound and Vibration, vol. 287, no. 1-2, pp. 129-151, 2005.

22. L. Guo, X. Zhang, and G. He, "Large-eddy simulation of circular cylinder flow at subcritical Reynolds number: Turbulent wake and sound radiation," Acta Mechanica Sinica, vol. 32, no. 1, pp. 1-11, 2016

23. O. Inoue and N. Hatakeyama, "Sound generation by a two- dimensional circular cylinder in a uniform flow," Journal of Fluid Mechanics, vol. 471, pp. 285-314, 2002.

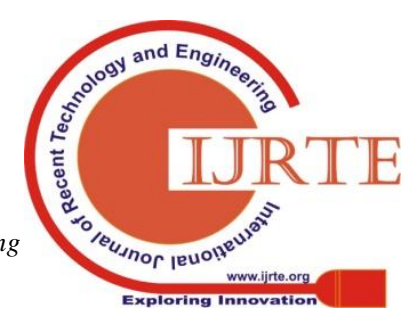


24. K. Karthik, S. Vengadesan, and S. K. Bhattacharyya, "Prediction of flow induced sound generated by cross flow past finite length circular cylinders," The Journal of the Acoustical Society of America, vol. 143 no. 1, pp. 260-270, 2018.

25. Y. Khalighi, A. Mani, F. Ham, and P. Moin, "Prediction of Sound Generated by Complex Flows at Low Mach Numbers," AIAA Journal, vol. 48, no. 2, pp. 306-316, 2010.

26. W. F. King and E. Pfizenmaier, "An experimental study of sound generated by flows around cylinders of different cross-section," Journal of Sound and Vibration, vol. 328, no. 3, pp. 318-337, 2009.

27. D. J. Moreau and C. J. Doolan, "Flow-Induced Sound of Wall- Mounted Finite Length Cylinders," AIAA Journal, vol. 51, no. 10, pp. 2493-2502, 2013

28. M. K. Chauhan, S. Dutta, B. S. More, and B. K. Gandhi, "Experimental investigation of flow over a square cylinder with an attached splitter plate at intermediate reynolds number," Journal of Fluids and Structures, 2018.

29. E. Latorre Iglesias, D. J. Thompson, and M. G. Smith, "Experimental study of the aerodynamic noise radiated by cylinders with different cross-sections and yaw angles," Journal of Sound and Vibration, vol. 361, pp. 108-129, 2016.

30. A. Purohit, A. K. Darpe, and S. P. Singh, "A study on aerodynamic sound from an externally excited flexible structure in flow," Computers and Fluids, vol. 103, pp. 100-115, 2014.

31. S. R. L. Samion, M. S. M. Ali, A. Abu, C. J. Doolan, and R. Z.

32. Y. Porteous, "Aerodynamic sound from a square cylinder with a downstream wedge," Aerospace Science and Technology, vol. 53, pp. 85-94, 2016

33. M. Sukri Mat Ali, C. J. Doolan, and V. Wheatley, "The sound generated by a square cylinder with a splitter plate at low Reynolds number," Journal of Sound and Vibration, vol. 330, no. 15, pp 3620-3635, 2011

34. H. Choi, W.-P. Jeon, and J. Kim, "Control of Flow Over a Bluff Body," Annual Review of Fluid Mechanics, 2008.

35. [34] M. Gad-el-Hak, "Flow Control," Applied Mechanics Reviews, 2013.

36. M. M. Zdravkovich, "Review and classification of various aerodynamic and hydrodynamic means for suppressing vortex shedding," Journal of Wind Engineering and Industrial Aerodynamics, 1981.

37. S. S. Collis, R. D. Joslin, A. Seifert, and V. Theofilis, "Issues in active flow control: Theory, control, simulation,' and experiment," Progress in Aerospace Sciences. 2004.

38. F. R. Grosche and G. E. A. Meier, "Research at DLR Göttingen on bluff body aerodynamics, drag reduction by wake ventilation and active flow control," Journal of Wind Engineering and Industrial Aerodynamics, 2001.

39. S. Rashidi, M. Hayatdavoodi, and J. A. Esfahani, "Vortex shedding suppression and wake control: A review," Ocean Engineering, vol. 126, no. October 2017, pp. 57-80, 2016.

40. C. J. Apelt, G. S. West, and A. A. Szewczyk, "The effects of wake splitter plates on the flow past a circular cylinder in the range lo4 $<\mathrm{R}$

41. < 5 x lo4," J . Pluid Me\&, vol. 61, no. 1, pp. 187-198, 1973.

42. R. Abdi, N. Rezazadeh, and M. Abdi, "Reduction of fluid forces and vortex shedding frequency of a circular cylinder using rigid splitter plates," European Journal of Computational Mechanics, vol. 26, no. 3, pp. 225-244, 2017.

43. M. Amiraslanpour, J. Ghazanfarian, and S. E. Razavi, "Drag suppression for 2D oscillating cylinder with various arrangement of splitters at $\mathrm{Re}=100$ : A high-amplitude study with OpenFOAM," Journa of Wind Engineering and Industrial Aerodynamics, vol. 164, no. February, pp. 128-137, 2017.

44. A. Nakayama and H. Noda, "LES simulation of flow around a bluff body fittted with a splitter plate," Journal of Wind Engineering and Industrial Aerodynamics, vol. 85, pp. 85-96, 2000.

45. [43] D. You, H. Choi, M.-R. Choi, and S.-H. Kang, "Control of Flow-Induced Noise Behind a Circular Cylinder Using Splitter Plates,' AIAA Journal, vol. 36, no. 11, pp. 1961-1967, 1998.

46. J. Y. Hwang and K. S. Yang, "Drag reduction on a circular cylinder using dual detached splitter plates," Journal of Wind Engineering and Industrial Aerodynamics, 2007.

47. K. Karthik, M. Vishnu, S. Vengadesan, and S. K. Bhattacharyya, "Optimization of bluff bodies for aerodynamic drag and sound reduction using CFD analysis," Journal of Wind Engineering and Industrial Aerodynamics, vol. 174, no. December 2017, pp. 133-140, 2018.

48. H. Akilli, C. Karakus, A. Akar, B. Sahin, and N. F. Tumen, "Control of Vortex Shedding of Circular Cylinder in Shallow Water Flow Using an Attached Splitter Plate," Journal of Fluids Engineering, vol. 130, no. 4, p. 041401, 2008.

49. K. Kwon and H. Choi, "Control of laminar vortex shedding behind a circular cylinder using splitter plates," Physics of Fluids, vol. 8, no. 2, pp. 479-486, 1996
50. [48] Y. Nakamura, "Vortex shedding from bluff bodies with splitter plates," Journal of Fluids and Structures, vol. 10, no. 2, pp. 147-158, 1996.

51. A. R. Ogunremi and D. Sumner, "The effect of a splitter plate on the flow around a finite prism," Journal of Fluids and Structures, vol. 59, pp. $1-21,2015$.

52. C. J. Doolan, "Flat-Plate Interaction with the Near Wake of a Square Cylinder," AIAA Journal, vol. 47, no. 2, pp. 475-479, 2009.

53. J. E. F. Williams and D. L. Hawkings, "Sound Generation by Turbulence and Surfaces in Arbitrary Motion," Philosophical Transactions of the Royal Society A: Mathematical, Physical and Engineering Sciences, 1969.

54. K. Deb, A. Pratap, S. Agarwal, and T. Meyarivan, "A fast and elitist multiobjective genetic algorithm: NSGA-II," IEEE Transactions on Evolutionary Computation, 2002.

55. J. Kennedy and R. Eberhart, "Particle swarm optimization," Neural Networks, 1995. Proceedings. IEEE International Conference on, 1995

56. R. Badhurshah, P. Dudhgaonkar, P. Jalihal, and A. Samad, "High efficiency design of an impulse turbine used in oscillating water column to harvest wave energy," Renewable Energy, vol. 121, pp. 344-354, 2018

57. J.-H. J.-H. Kim, B. Ovgor, K.-H. Cha, J.-H. J.-H. Kim, S. Lee, and K.-Y. Kim, "Optimization of the Aerodynamic and Aeroacoustic Performance of an Axial-Flow Fan,” AIAA Journal, vol. 52, no. 9, pp. 2032-2044, 2014.

58. L. W. Nogueira and B. S. Carmo, "Numerical analysis and acoustic optimization of a detached splitter plate applied for passive cylinder wake control," Journal of the Brazilian Society of Mechanical Sciences and Engineering, vol. 40, no. 1, pp. 1-16, 2018.

59. H. Liu, M. Azarpeyvand, J. Wei, and Z. Qu, "Tandem cylinder aerodynamic sound control using porous coating," Journal of Sound and Vibration, vol. 334, pp. 190-201, 2015.

60. M. R. Khorrami, M. M. Choudhari, D. P. Lockard, L. N. Jenkins, and C. B. Mcginley, "Unsteady Flowfield Around Tandem Cylinders as Prototype Component Interaction in Airframe Noise," AIAA Journal, 2007

61. F. R. Menter, "Two-equation eddy-viscosity turbulence models for engineering applications," AIAA Journal, 1994.

62. D. C. D. C. D. C. Wilcox, "Turbulence Modeling for CFD: Text," Aiaa. 2006

63. B. P. Leonard, "A stable and accurate convective modelling procedure based on quadratic upstream interpolation," Computer Methods in Applied Mechanics and Engineering, 1979.

64. R. Porteous, D. J. Moreau, and C. J. Doolan, "The aeroacoustics of finite wall-mounted square cylinders," Journal of Fluid Mechanics, vol. 832, no. September, pp. 287-328, 2017.

65. B. J. Vickery, "Fluctuating lift and drag on a long cylinder of square cross-section in a smooth and in a turbulent stream," Journal of Fluid Mechanics, vol. 25, no. 3, pp. 481-494, 1966.

66. X. Tian, M. C. Ong, J. Yang, and D. Myrhaug, "Unsteady RANS simulations of flow around rectangular cylinders with different aspect ratios," Ocean Engineering, 2013.

67. G. Bosch and W. Rodi, "Simulation of vortex shedding past a square cylinder with different turbulence models," International Journal for Numerical Methods in Fluids, 1998.

68. K. Shimada and T. Ishihara, "Application of a modified $k-\varepsilon$ model to the prediction of aerodynamic characteristics of rectangular cross- section cylinders," Journal of Fluids and Structures, 2002

69. S. Murakami and A. Mochida, "On turbulent vortex shedding flow past 2D square cylinder predicted by CFD," Journal of Wind Engineering and Industrial Aerodynamics, 1995.

70. A. Sohankar, "Flow over a bluff body from moderate to high Reynolds numbers using large eddy simulation," Computers and Fluids, vol. 35, no. 10, pp. 1154-1168, 2006.

71. J. H. Park, "A laser-Doppler velocimetry study of ensemble- averaged characteristics of the turbulent near wake of a square cylinder," Journa of Fluid Mechanics, vol. 304, pp. 285-319, 1995.

72. P. D. Welch, "The Use of Fast Fourier Transform for the Estimation of Power Spectra: A Method Based on Time Averaging Over Short, Modified Periodograms," IEEE Transactions on Audio and Electroacoustics, 1967.

73. A. V Oppenheim and R. W. Schafer, "Discrete Time Signal Processing," Book. 2009.

74. C. J. Apelt, G. S. West, and A. A. Szewczyk, "The effect of wake splitter plates on the flow past a circular cylinder in the range of $10000<\mathrm{R}<50000$," Journal of Fluid Mechanics, vol. 61, no. 1973, pp 187- 198, 1973.

75. D. A. Pandya, B. H. Dennis, and R. D. Russell, "A computational fluid dynamics based artificial neural network 
model to predict solid particle erosion," Wear, 2017.

76. Z. Wei, Z. Yang, C. Xia, and Q. Li, "Journal of Wind Engineering \& Industrial Aerodynamics Cluster-based reduced-order modelling of the wake stabilization mechanism behind a twisted cylinder," Journal of Wind Engineering \& Industrial Aerodynamics, vol. 171, no. November, pp. 288-303, 2017.

77. R. Li, P. Xu, Y. Peng, and P. Ji, "Journal of Wind Engineering Multi-objective optimization of a high-speed train head based on the FFD method," J. of Wind Engineering and Industrial Aerodynamics, vol. 152 , pp. 41-49, 2016.

\section{AUTHORS PROFILE}

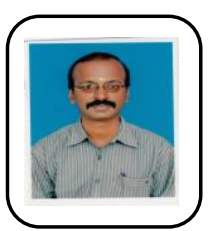

Dr. S. Jeyakumar working as Professor in Aeronautical Engineering, Kalasalingam Academy of Research and Education. The author has published papers in the areas of high-speed flow mixing and combustion, cavity flow, dual combustion ramjet engine.

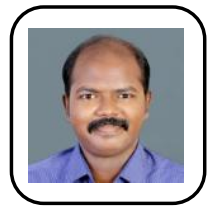

K. Karthik working as Assistant Professor in Aeronautical Engineering, Kalasalingam Academy of Research and Education. The author has published papers in the areas of CFD, and aero acoustics.

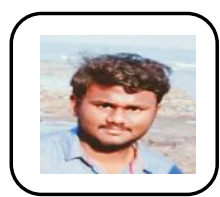

Ramesh Babu S was a student of Kalasalingam Academy of Research and Education and is currently working as Design engineer in Bangalore, India 